\title{
SUBSTRATE DEFECTS FILTRATION DURING EPITAXIAL LATERAL OVERGROWTH OF GaAs
}

\author{
Z.R. ŻYTKIEWICZ AND D. DoBosZ \\ Institute of Physics, Polish Academy of Sciences \\ Al. Lotników 32/46, 02-668 Warsaw, Poland
}

\begin{abstract}
Results on the growth of GaAs on (001) GaAs substrates by the epitaxial lateral overgrowth technique are reported. We show that the ratio of normal to lateral growth rates in the epitaxial lateral overgrowth process can be controlled by the crystallographic orientation of the seeds and by $\mathrm{Si}$ adding to the melt. Experimental data showing that the dislocations threading from the substrate are efficiently filtered and cannot propagate to the epitaxial lateral overgrowth layers are presented. These findings prove that the epitaxial lateral overgrowth process is the powerful method to grow epilayers with low dislocation density on high dislocation density substrates.
\end{abstract}

PACS numbers: 68.55.Ln

There is a continuous effort to reduce the defect density in heteroepitaxial structures grown on lattice mismatched substrates. Very sophisticated techniques of buffer layers and of defects filtration by superlattices have been elaborated to improve the quality of epitaxial layers thicker than the critical thickness. Unfortunately, very often the number of defects is still unacceptably high. Therefore, the growth of dislocation free epitaxial layers remains one of the most important goals in heteroepitaxy. The very promising way to reduce the dislocation density in heteroepitaxy is the epitaxial lateral overgrowth (ELO) technique when combined with liquid phase epitaxy (LPE) growth method. However, controversy exists about the growth mechanism and the defect structure in layers grown by ELO. In this work we report the results on the growth of GaAs epilayers on (001) GaAs substrates using the ELO method. The growth mechanism and the effect of substrate defects filtration during the ELO procedure are discussed.

The principle of the epitaxial lateral overgrowth technique is shown schematically in Fig. 1. The substrate was covered by a $0.1 \mu \mathrm{m}$ thick $\mathrm{SiO}_{2}$ film and patterned by a conventional photolithography to form on its whole area a grating of $10 \mu \mathrm{m}$ wide oxide-free seeding windows. Then, an epitaxial layer was deposited on such substrate by the LPE technique. In our experiments the growth was initiated by covering the substrate with the saturated $\mathrm{Ga}-\mathrm{As}$ solution at the temperature of $750^{\circ} \mathrm{C}$ while cooling the system. GaAs nucleated on the line seeds and the growth proceeded in the direction normal to the substrate. Then, the growth in lateral 
direction over the oxide film started when the crystallization front exceeded the top layer of $\mathrm{SiO}_{2}$. The key feature of the ELO technique is a distribution of defects in the grown layers. It is known that dislocations present in the substrate cannot penetrate through the polycrystalline $\mathrm{SiO}_{2}$ film. If propagation of dislocations from the substrate is solely a source of defects, they should be present in the epitaxial layer only in the areas grown vertically over the seeds (see Fig. 1). Therefore, a significant reduction of an average defect density in the layer is expected if its horizontal dimension $L$ is larger than the width of the growth window. GaAs nucleation on the $\mathrm{SiO}_{2}$ film must be avoided for a successful ELO. In the LPE growth method supersaturation of a solution is low and this requirement can be easily fulfilled. On the contrary, in MBE or MOCVD techniques polycrystalline growth on the mask usually takes place $[1,2]$ making these methods useless for the ELO growth.

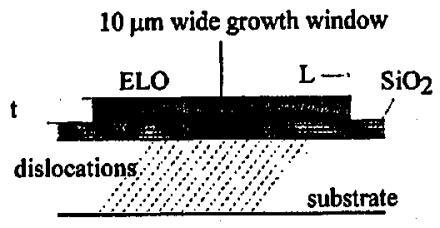

Fig. 1. Schematic cross-section of the ELO showing the principle of substrate defects filtration.
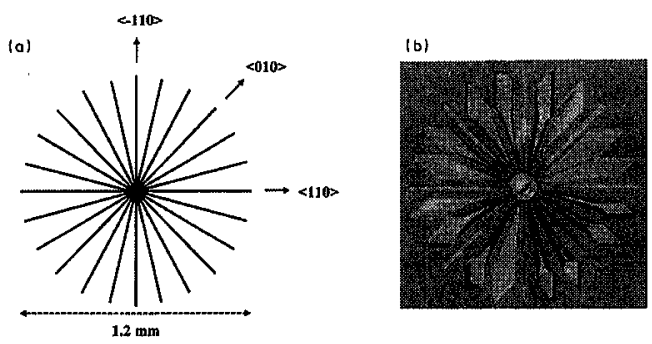

Fig. 2. Schematic diagram of the star-like seed pattern (a) and a microphotograph of the ELO grown from radial line seed (b). The line seeds were $10 \mu \mathrm{m}$ wide.

The crucial point of the ELO technique is to find the growth conditions under which the value of the aspect ratio $L / t$ is the largest. Then, the filtration of substrate defects should be the most effective. It has been suggested [3] that the ratio of lateral to normal growth velocities can be controlled by varying the crystallographic orientation of the line seeds in the plane of the substrate. The basic idea behind this is to find such orientation of the stripe for which the side walls of the ELO layer are covered by atomically rough, fast growing planes, while the slowly grown facet is formed on the upper surface. We checked this possibility by using GaAs substrate with the seeds of star-like pattern (see Fig. 2a) on which GaAs layer was deposited by the LPE. 

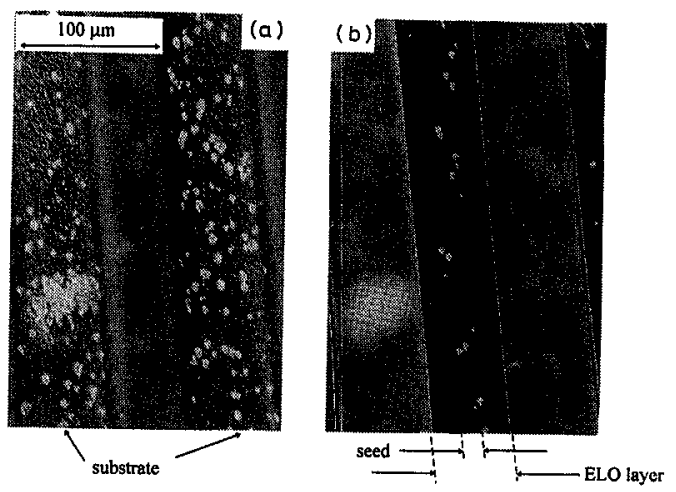

Fig. 3. Microphotograph of GaAs ELO layer grown on the substrate with the parallel line seeds. The layer was etched in molten $\mathrm{KOH}$ in order to show the distribution of etch pits in the substrate (a) and in the ELO layer (b). Notice that the etch pits in the layer are visible only over the line seed.

Figure 2b shows the overgrowth of a star-like pattern on a (001)-oriented GaAs substrate. The growth was forced by cooling the system with the rate of $0.2^{\circ} \mathrm{C} / \mathrm{min}$ for 50 minutes. As it is seen in the picture, the width of ELO layers increases with the distarce from the centre of the pattern. This indicates that the supersaturation of the solution was not homogeneous over the whole pattern. Indeed, the supersaturation should be low in the centre of the star. This is because a high density of line seeds there allows for a very efficient consumption of the solute. On the other hand, the density of places a vailable for nucleation is low on the periphery of the pattern. Therefore, the stream of As from the bulk of the solution was significantly increased by the diffusion of solute from the outside of the patterned area. This led to the increase in the supersaturation of the solution and to a faster lateral growth in the areas far away from the centre of the pattern.

From Fig. $2 \mathrm{~b}$ the dependence of lateral growth rate on the orientation of the seeds can be easily found. The width of the ELO was the largest when the stripe was $15^{\circ}$ and $30^{\circ}$ off-oriented from the $\langle 110\rangle$ and $\langle 010\rangle$ directions, or their equivalent orientations, while it is the smallest for the $\langle 110\rangle$ and $\langle 010\rangle$ directions. From the simple geometrical considerations the facets on the side walls of ELO layers were identified as the slowly grown $\{111\}$ and $\{100\}$ planes. Their formation which proceeded much faster when the stripe is oriented in the $\langle 110\rangle$ and $\langle 010\rangle$ directions, slowed down the lateral development of the ELO layer. Therefore, the line seeds oriented $15^{\circ}$ or $30^{\circ}$ off from the $\langle 110\rangle$ or $\langle 010\rangle$ directions should be used to obtain a large value of the lateral to normal growth rate ratio.

According to the above discussion, we have chosen the orientation $15^{\circ}$ off from the $\langle 110\rangle$ direction to grow ELO layers on the (001) GaAs substrate with the parallel line seeds. The width and the distance between the growth windows were $10 \mu \mathrm{m}$ and $100 \mu \mathrm{m}$, respectively. Figure 3 is a photograph of the layer grown at $750^{\circ} \mathrm{C}$ by cooling the system with the rate of $0.5^{\circ} \mathrm{C} / \mathrm{min}$ for 40 minutes. The ELO layer was $8 \mu \mathrm{m}$ thick and $58 \mu \mathrm{m}$ wide, which corresponded to the value of 
the aspect ratio $L / t=3$. The layer was etched after the growth in molten $\mathrm{KOH}$ at $346^{\circ} \mathrm{C}$ for 3 minutes. Such procedure is commonly used to visualise distribution of dislocations in GaAs [4]. First, it is noteworthy that the side walls of the layers shown in Fig. 3b, are smooth and no low index planes limiting the lateral growth were formed. This indicates that the side faces of the layers were the fast growing atomically rough planes and much wider layers could be obtained if the growth was continued. The main result of this work becomes visible from Fig. 3 when the etch pits distributions in the substrate (Fig. 3a) and in the ELO layer (Fig. 3b) are compared. The etch pits density (EPD) in the substrate was very high $\left(5 \times 10^{4} \mathrm{~cm}^{-2}\right)$, which was in agreement with the supplier specification of substrates used. On the contrary, in the ELO layers only the dislocations threading from the substrate were observed. As shown in Fig. 3b, density of these dislocations was low and they were confined in a very narrow area above the seed. The rest of the ELO layer is dislocation free which was additionally proved by the studies of the ELO layers with the use of cathodoluminescence technique. This finding shows that the mechanism of substrate defects filtration active during ELO of GaAs was very efficient and proves the correctness of the schema shown in Fig. 1.

We have grown wider the dislocation-free parts of ELO layers by adding 1 at.\% of Si to the Ga-As solution. This step improved morphology of the layers. Moreover, the growth rate in the direction normal to the substrate was reduced which can be explained in terms of step flow retardation by impurity atoms on atomically flat upper surface of the layer [5]. However, the lateral growth rate was not affected by doping. Therefore, for the layers doped with Si we were able to increase the ratio of lateral to normal growth rate to the value of 5 . Because the dislocation distribution in the $L=45 \mu \mathrm{m}$ wide and $t=9 \mu \mathrm{m}$ thick layer was similar to that shown in Fig. $3 \mathrm{~b}$, simple geometrical calculations show that nearly $90 \%$ of the layer area was dislocation free.

Summarising the paper, we have shown that the substrate defects are effectively filtered during the LPE ELO growth of GaAs. The dislocations threading from the substrate are observed only above the growth window leaving the rest of the layer (i.e. up to $90 \%$ of its area) dislocation free. By proper adjustment of the growth parameters wide ELO layers with a smooth upper surface were obtained. Finally, it is worth mentioning that by reducing the cooling rate to $0.1^{\circ} \mathrm{C} / \mathrm{min}$ and increasing the growth time to 200 minutes a full coverage of the substrate with the Si doped GaAs ELO layers was obtained.

This work is partly supported by the Committee for Scientific Research grant No. 8 T11B 03010.

\section{References}

[1] R.J. Matyi, H. Shichijo, T.M. Moore, H-L. Tsai, Appl. Phys. Lett. 51, 18 (1987).

[2] P. Vohl, J. Cryst. Growth 54, 101 (1981).

[3] T. Nishinaga, Cryst. Prop. Prep. 31, 92 (1991).

[4] G. Nozle, V. Geist, G. Wagner, P. Paufler, K. Jurkschat, Z. Kristallogr. 193, 111 (1990).

[5] M. Ohara, R.C. Reid, Modeling Crystal Growth Rates from Solution, Prentice-Hall, Englewoods Cliffs 1973. 